\title{
High Sensitivity, High Selectivity SERS Detection of MnSOD Using Optical Nanoantennas Functionalized with Aptamers
}

\author{
Maximilien Cottat ${ }^{a, 1}$, Cristiano D'Andrea ${ }^{b, 1}$, Ryohei Yasukuni ${ }^{a}$, Natalia Malashikhina $^{c}$, \\ Ruta Grinyte ${ }^{c}$, Nathalie Lidgi-Guigui ${ }^{a}$, Barbara Fazio ${ }^{b}$, Angela Sutton ${ }^{d}$, Olivier Oudar ${ }^{d}$, \\ Nathalie Charnaux ${ }^{d}$, Valery Pavlov ${ }^{c}$, Andrea Toma ${ }^{e}$, Enzo Di Fabrizio ${ }^{f, 2}$, Pietro G. \\ Gucciardi ${ }^{b, *}$, Marc Lamy de la Chapelle ${ }^{a, * *}$
}

${ }^{a}$ Université Paris 13, Sorbonne Paris Cité, Laboratoire CSPBAT, CNRS, (UMR 7244), 74 rue Marcel Cachin, F-93017 Bobigny, France

${ }^{b}$ CNR IPCF Istituto per i Processi Chimico-Fisici, Viale F. Stagno D'Alcontres 37, I-98156, Messina, Italy

${ }^{c}$ Biofunctional Nanomaterials Department, CIC biomaGUNE, Parque tecnológico de San Sebastian, Paseo Miramon 182, Donostia- San Sebastian 20009, Spain

${ }^{d}$ Université Paris 13, Sorbonne Paris Cité, Bioingénierie cardiovasculaire, INSERM, (U1148), rue Marcel Cachin, 93017, Bobigny, France

${ }^{e}$ Istituto Italiano di Tecnologia. Via Morego 30, 16163 Genova, Italy

${ }^{f}$ University of Magna Graecia, BIONEM lab, Campus Salvatore Venuta, Viale Europa 88100 Germaneto-Catanzaro, Italy

${ }^{1}$ These authors have contributed equally to the work presented in this publication

${ }^{2}$ Present address : KAUST, King Abdullah University of Science and Technology, Physical Science and Engineering and BESE Divisions, Thuwal, 23955 -6900, Kingdom of Saudi Arabia

* Corresponding author : fax: +39-090-39.74.130

** Corresponding author at: University Paris 13, Laboratoire CSPBAT, 74 rue Marcel Cachin, 93017 Bobigny, France. Tel: +33148387691

E-mail adresses : gucciardi@its.me.cnr.it (P.G. Gucciardi) and marc.lamydelachapelle@univparis13.fr (M. Lamy de la Chapelle) 
Keywords: SERS, biosensor, plasmon resonance, MnSOD, biomarker, nanoantenna, aptamers

\begin{abstract}
In this paper, we present the development of a highly sensitive, specific and reproducible nanobiosensor to detect one specific liver cancer biomarker, the Manganese Super Oxide Dismutase (MnSOD). The high sensitivity and reproducibility was reached by using SERS on gold nanostructures (nanocylinders and coupled nanorods) produced by electron-beam lithography (EBL). The specificity of the detection was provided by the use of a specific aptamer with high affinity to the targeted protein as a recognition element. With such sensor, we have been able to observe the SERS signal of the MnSOD at concentrations down to the nM level and to show with negative control that this detection is specific due to the use of the aptamer. This latter issue has allowed us to detect the MnSOD in different body fluids (serum and saliva) at concentrations in the $\mathrm{nM}$ range. We have then demonstrated the effectiveness of our SERS nanobiosensor using aptamer as a bioreceptor for the detection of disease biomarker at low concentration and in complex fluids.
\end{abstract}




\section{INTRODUCTION}

Biosensors development is a very active research field since it will pave the way to new detection methods with improved characteristics (better sensitivity, label free detection, better selectivity...) and since it can have a wide range of applications especially in the medical field such as the disease diagnosis, prognosis or monitoring. Different electrochemical, ${ }^{1,2}$ optical $^{3-8}$ and mechanical ${ }^{9-11}$ technologies have been developed with success to detect biological agents, such as microbial toxins ${ }^{12}$ and pathogens. ${ }^{13,14}$ However, even if such techniques proposed improvements and new detection strategies, some major drawbacks can be noticed. The fluorescent techniques need a labeling of the molecules to be detected, which could induce a change in the biomolecule activity and thus a bias in the detection. Quartz crystal microbalance $(\mathrm{QCM}),{ }^{11}$ surface plasmon resonance $(\mathrm{SPR})^{8}$ or Enzyme-linked immunoassay arrays (ELISA) ${ }^{1}$ methods, can only permit the detection of the molecule when interacting with the biosensor surface but not its direct identification. The identification, in fact, is done thanks to the use of a bioreceptor at the sensor surface characterized by a high affinity with the targeted biomolecules but that cannot avoid a non-specific interaction that gives rise to a signal noise that affects the lower limit of detection (LOD) typically estimated between $10^{-9} \mathrm{M}$ to $10^{-11} \mathrm{M}$, depending on the affinity constant of the bioreceptor to the targeted analyte. This drawback can be overcome by the design of a bioreceptor having higher affinity than the standard antibodies, using, as example DNA aptamers, ${ }^{15}$ or by the exploiting of spectroscopic detection methods such as Raman spectroscopy, ${ }^{16}$ that can provide a direct identification of the molecule bound to the bioreceptor, ${ }^{14}$ and then distinguish the specific analyte from the non-specific ones. 
Aptamers are DNA strands that exhibit a higher affinity to proteins than antibodies, they have become increasingly important molecular tools for diagnostics and therapeutics since they can be synthesized with high reproducibility and purity from commercial sources, showing a high chemical stability. ${ }^{14,17-21}$ Raman spectroscopy $(\mathrm{RS})^{22}$ provides a label free spectral signature of the analyte and represents the right candidate as vibrational technique for a direct identification of the bio-analytes. ${ }^{16,23}$ Despite the Raman cross section is very low compared to fluorescence (more than 10 orders of magnitude) its sensibility can be improved by exploiting the Surface Enhanced Raman Scattering (SERS) effect. ${ }^{24,25}$ The principle of SERS is based on the strong interaction between metallic nanoparticles and light (i.e plasmon resonance, lightning rod effect...) that induces a high local enhancement of the electromagnetic field at the nanoparticle vicinity. ${ }^{26}$ The nanoparticle, in turn, acts as an optical nanoantenna enhancing the Raman scattering of any molecules deposited at the nanoparticle surface, ${ }^{27}$ thus providing a total Raman signal amplification of several orders of magnitude (up to $10^{9}$ ), ${ }^{28}$ paving the way to the single molecule sensitivity. ${ }^{29,}, 30$

Several SERS biosensors architectures have been proposed in the literature. ${ }^{31-35}$ Colloidal nanoparticles permit to achieve the detection of the P38 mitogen-activated protein kinase $^{36}$ down to $10^{-11} \mathrm{M}$, being also effective for the detection of biomolecules in body fluids. The detection of folic acid in human serum ${ }^{37}$ has been demonstrated down to $10^{-8} \mathrm{M}$, as well as the detection of prostate-specific antigen in serum was achieved down to $\mathrm{fM}$ concentrations $(\sim 1 \mathrm{pg} / \mathrm{mL}) .{ }^{38}$ Unfortunately, these kinds of SERS biosensor are affected by several drawbacks that can compromise such systems. Indeed, the colloidal nanoparticles often aggregate together inducing a change in the SERS enhancement and influencing the reliability of the detection. Such 
technique is also restricted to water-soluble nanoparticles or molecules. To overcome these disadvantages, the design of well-controlled SERS substrates produced by lithographic methods have been proposed. ${ }^{34}$

Nanosphere lithography (NSL) or Electron Beam Lithography (EBL) are well known to provide nanostructures with good controlled size, shape and spacing parameters at the nanometric scale, allowing the control and tuning of the localized plasmon resonances and the optimization of the SERS efficiency. ${ }^{39,40} 41,42$ Van Duyne's group has demonstrated that sensors produced by NSL are suitable for the detection of glucose, ${ }^{43}$ also in vivo, ${ }^{44}$ or bacillus anthracis. ${ }^{45}$ SERS substrates produced by EBL permitted the detection of pure proteins like as Bovine Serum Albumine (BSA), lysozym, RNase, myoglobin at concentrations down to the attomolar. $^{46,47}$ The possibility to functionalize colloidal metal nanoparticles with aptamers so to obtain a highly specific SERS sensor has been demonstrated on trombin (LOD of $1 \mathrm{nM}$ in presence of complex biofluids), ${ }^{48}$ adenosine triphosphate (LOD of $12.4 \mathrm{pM}),{ }^{49}$ vasopressin (LOD on the order of $5.2 \mu \mathrm{U} / \mathrm{mL}$ ). ${ }^{50}$ These experimental demonstrations in addition to predictions related to a better optimization of the limit of detections clearly show that the SERS spectroscopy can be a very powerful tool for the detection of biomolecules. However, the binding between the targeted molecules and the bioreceptor is crucial to catch the analyte close to the surface and thus to enhance its Raman signature. The sensitivity of the detection is then related to both the high enhancement induced by the nanoparticles and the affinity of the bioreceptor with the analyte. To reach the lowest detection limit, one the most significant parameters is to have an optimized SERS substrate that provides the highest enhancement factor and a bioreceptor exhibiting a high affinity with the analytes in order to have higher probability to capture them at the nanostructure 
surface. Moreover, since the identification is done thanks to the specific spectral signature of analytes, the detection is not done only through the biorecognition event induced by the bioreceptor but also with the Raman spectrum. This implies that the detection is not disturbed by nonspecific interaction with the bioreceptor (that will be directly identified using the SERS).

For a complete affirmation of the SERS spectroscopy as new viable alternative tool, however, detection of a larger class of biomarkers is necessary proving that detection thresholds can be better with respect to the best ones reached by the classical techniques as ELISA (tipically $10^{-6}-10^{-8} \mathrm{M}$ ) or high-performance liquid chromatography fluorescence (HPLC) $\left(10^{-7} \mathrm{M}\right)$.

Manganese superoxide dismutase (MnSOD or SOD2) is one of the primary antioxidant enzymes, capable to maintain intracellular reactive oxygen species (ROS) and redox balance for the cell equilibrium, while protecting normal tissue against oxidative stress. $^{51,52}$ Mukhopadhyay et al. observed an increased expression of MnSOD gene in cancerous cell lines. ${ }^{53}$ Increased activity of MnSOD and of its levels in sera have also been correlated to severe chronic liver diseases and can lead to enhance malignant transformation inducing the development of various carcinoma. ${ }^{57-}$ ${ }^{60}$ The measurement of the MnSOD concentration in body fluids is then of primarily importance to monitor the clinical course and also the stages of tumors ${ }^{61}$ and provide a diagnosis and a prognosis of the disease at its earliest stage. The most common method used for the MnSOD detection is the immuno-assay and more especially the ELISA test. Under optimum conditions, the sensitivity of ELISA assays permit the detection of 2-200 $\mathrm{ng}\left(10^{-5}-10^{-7} \mathrm{~mol} / \mathrm{l}\right)$ of purified MnSOD. The detection threshold of such technique is thus in the range of the micromolar. Moreover, the ELISA is a 
highly time-consuming technique since it is necessary to wait for several hours to obtain a quantitative result.

In this study, we present a new high specificity, high sensitivity SERS nanobiosensor for the detection of MnSOD. The nanobiosensor combines the high sensitivity offered by gold nanoantennas produced by $\mathrm{EBL}^{39,62}$ with the high specificity offered by the functionalization of the sensor surface with thiolated aptamers. We demonstrate that thanks to the large enhancement of the local Raman radiation we are able to accomplish label free detection of MnSOD with nanomolar sensitivity in addition to a high selectivity guaranteed by a functionalization with aptamers specifically designed to capture a target protein. We prove the high sensitivity of our nanobiosensor concept by detecting pure protein solutions with nanoantennas featuring two different shapes, namely nanorods dimers and individual nanocylinders, and using two excitation wavelengths in the visible (660nm) and in the NIR (785nm). We validate the specificity of our biosensor with negative controls on BSA. Finally, we demonstrate the detection of this MnSOD at low concentration in two different body fluids, such as sera and saliva.

\section{RESULTS AND DISCUSSION}

\section{Aptamers for MnSOD.}

Potentially, three different recognition elements for detection of MnSOD could be employed for fabrication of affinity assays: antibodies, $F\left(a b^{\prime}\right)_{2}$ fragments of antibodies and DNA aptamers. Antibodies are comparatively larger then $F\left(a b^{\prime}\right)_{2}$ fragments and DNA aptamers. The size of an antibody is close to $5 \mathrm{~nm}$ whereas it is around $2 \mathrm{~nm}$ for a $\mathrm{F}\left(\mathrm{ab}^{\prime}\right)_{2}$ and even lower for an aptamer (estimated to be close to 1 $\mathrm{nm})$. Analytes captured through antibodies on the surface on nanoantennas would then stay too far away from the metal surface for efficient amplification of the Raman 
signal resulting in loss of sensitivity. $\mathrm{F}\left(\mathrm{ab}^{\prime}\right)_{2}$ fragments are smaller than the whole antibodies inducing a lower distance between the analyte and the nanoantenna surface. But the procedure for generation of $\mathrm{F}\left(\mathrm{ab}^{\prime}\right)_{2}$ fragments by breaking the S-S bonds leaves them partially denatured. Therefore, each batch of $\mathrm{F}\left(\mathrm{ab}^{\prime}\right)_{2}$ fragments shows different apparent affinity constants. The production of DNA with DNA synthesizer is much more convenient and reproducible than the cleavage of antibodies to produce $\mathrm{F}\left(\mathrm{ab}^{\prime}\right)_{2}$ fragments. On another hand, each batch of DNA aptamers produced with a DNA synthesizer has equal affinity to their target molecules, hence DNA aptamers were employed in this work.

Thiolated aptamers specific for MnSOD (5'- HS- (-O- $\left.\mathrm{CH}_{2}-\mathrm{CH}_{2}-\mathrm{O}-\right)_{6}$ TT TTT TTT TTT TTT TTC TTC TCT AGC TGA ATA ACC GGA AGT AAC TCA TCG TTT CGA TGA GTT ACT TCC GGT TAT TCA GCT AGA GAA G-3’) are prepared by automated solid-phase oligonucleotide synthesis in a $1 \mu \mathrm{M}$ scale (see Methods). The success of the functionalization process is fundamental to maximize the specificity and the sensibility of our sensor. For this aim we used a quartz microbalance $(\mathrm{QCM})$ to determine the affinity of the aptamer to the MnSOD and the surface coverage. We have established a functionalization protocol, schematically illustrated in SI, Figure S1, divided in 4 steps that are applied for both QCM and subsequent SERS experiments on the nanoantennas (for more details see SI, S-2).

\section{Specific SERS detection of MnSOD}

Two different geometries of gold nanoantennas have been fabricated by EBL (see see Experimental Section) and exploited to test our nanobiosensor (1): dimers of nanorods (a) with length $L=100 \mathrm{~nm}$, width $w=60 \mathrm{~nm}$, height $h=50 \mathrm{~nm}$ and gap $G=20 \mathrm{~nm}$, and individual nanocylinders (b) with diameters $D=140 \mathrm{~nm}$, height of $50 \mathrm{~nm}$. The nanoantennas are arranged in arrays separated by $200 \mathrm{~nm}$ to one another in both 
directions of the plane. The diameters of the nanocylinders $(140 \mathrm{~nm})$ have been chosen so to match the excitation wavelength of $660 \mathrm{~nm}$ with the optimized position of the plasmon resonance and thus to reach the highest SERS enhancement. The nanorod dimers were designed to be excited at $785 \mathrm{~nm} .{ }^{62}$

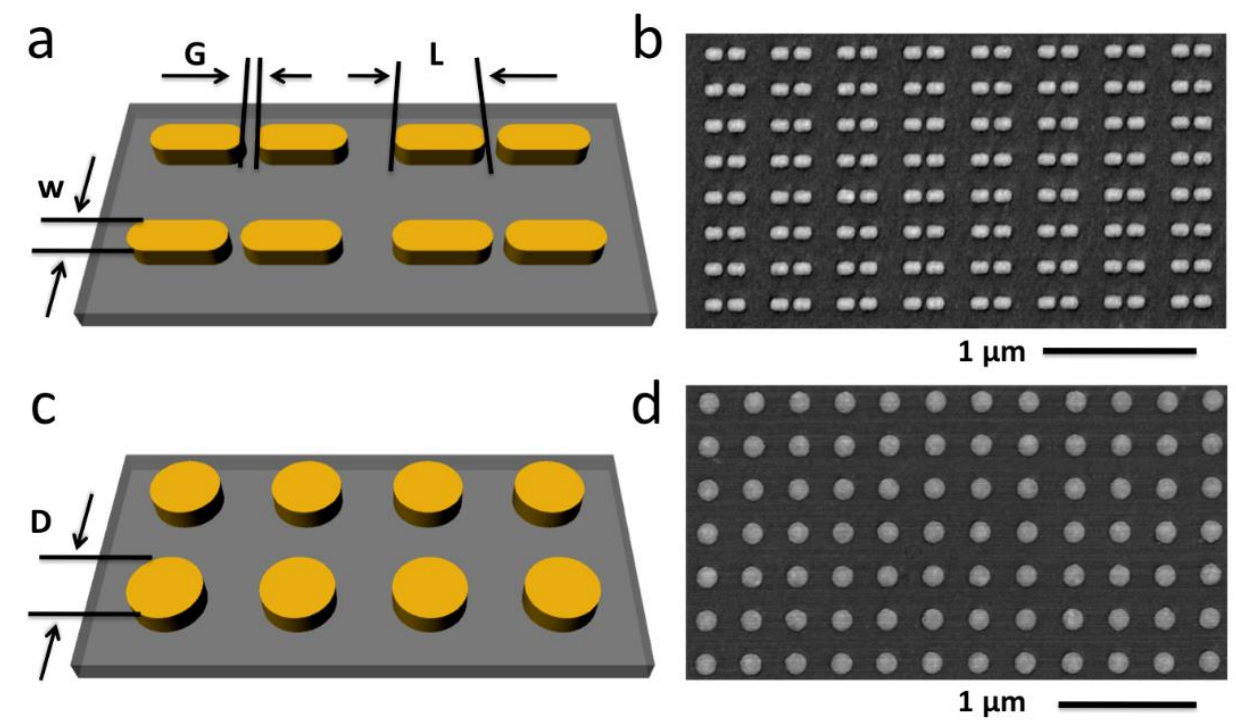

Figure 1: $(\mathrm{a}, \mathrm{c})$ Schematic illustrations of the two different plasmonic devices adopted in the present work. The characteristic geometrical parameters are reported (i.e. nanorods length $L$, width $w$ and gap $G$; nanocylinder diameter $D$ ). Representative scanning electron microscopy (SEM) images of the nanorod dimer arrays and nanocylinders are shown in $(b, d)$, respectively.

\section{Protein detection}

The nanoantennas have been functionalized following a protocol similar to that used for the QCM measurements (see Figure S1, for more details see SI, S-3). Considering an average surface close to $6 \mathrm{~nm}^{2}$ occupied by one aptamer, if we assume that the surface functionalization is similar for flat gold surface and gold nanostructures, we can estimate that a nanocylinder with a diameter of $140 \mathrm{~nm}$ is covered by $6 \times 10^{3}$ of aptamers whereas a nanorod dimer is covered by $7 \times 10^{3}$ aptamers. The target protein 
is finally captured by immersing the functionalized nanoantennas in a buffer solution (Tris- $\mathrm{HCl} 50 \mathrm{mM}, \mathrm{NaCl} 100 \mathrm{mM}, \mathrm{MgCl} 25 \mathrm{mM}, \mathrm{pH}$ 8.2) of $\mathrm{MnSOD}$ at various concentrations for $1 \mathrm{~h}$, followed by rinsing with buffer and drying under nitrogen flow.

SERS experiments are performed using an Xplora spectrometer from Horiba Scientific (see Methods). First measurements have been targeted at determining the SERS background on the bare nanoantennas ${ }^{63,64}$ and from the SERS generated by the functionalization layer (DNA, and DNA + 6-MOH). As shown in Figure 2 (black line), the signal from the bare antennas (after plasma ozone cleaning) is almost flat with only some vibration peaks of the $\mathrm{CaF}_{2}$ substrate appearing. ${ }^{65}$ Much stronger signal is observed on antennas coated with the aptamer (Figure 2, red line).

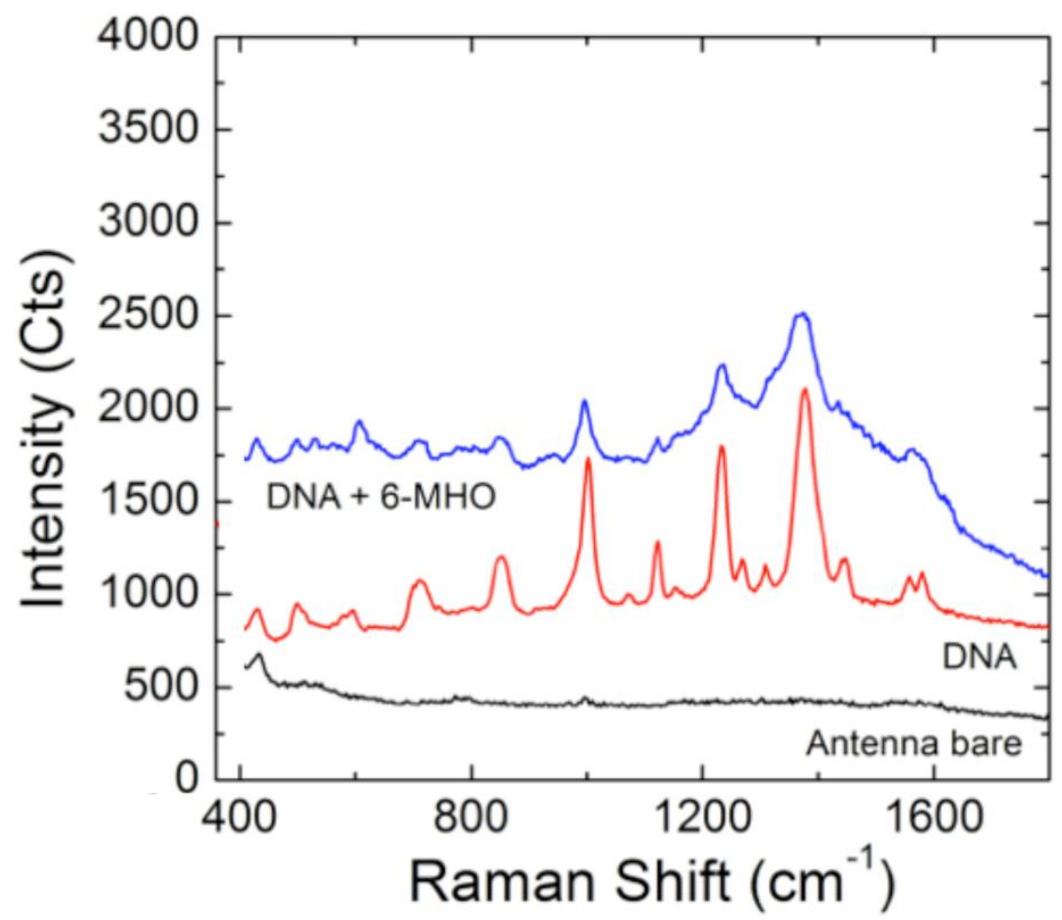

Figure 2: SERS spectra of the gold nanorods dimers $\left(\lambda_{\text {exc }}=785 \mathrm{~nm}\right)$ after ozone plasma cleaning (black line), after functionalization with thiolated DNA aptamers (red line) and after the addition of a further blocking layer of 6-MOH (blue line). Data are shown without any background subtraction. 
Strongest signal is observed at $1000 \mathrm{~cm}^{-1}$ (C-O stretch in deoxyribose), ${ }^{66,67} 1234 \mathrm{~cm}^{-1}$ (Thymine, Ring- $\mathrm{CH}_{3}$ Stretching), ${ }^{66,68-71}$ and $1380 \mathrm{~cm}^{-1}$ (Thymine, Guanine) ${ }^{66,67,69-73}$ with less intense peaks at and $715 \mathrm{~cm}^{-1}$ (Adenine), ${ }^{66,73-76} 850 \mathrm{~cm}^{-1}$ (sugar vibration), ${ }^{66}$ $1125 \mathrm{~cm}^{-1}\left(\mathrm{PO}_{2}\right.$ backbone $),{ }^{66,} 67$ and smaller contributions at $1075 \mathrm{~cm}^{-1}\left(\mathrm{PO}_{2}\right.$ backbone), ${ }^{66,67,75} 1234 \mathrm{~cm}^{-1}$ (Thymine), 66, 67, 70 1301 $\mathrm{cm}^{-1}$ (Cytosine), ${ }^{69,}$ 70, 74, 77 $1450 \mathrm{~cm}^{-1}$ (Cytosine, Thymine), ${ }^{67-69,78} 1556 \mathrm{~cm}^{-1}$ (Adenine), ${ }^{68,78} 1580 \mathrm{~cm}^{-1}$ (Adenine, Guanine). ${ }^{66,68,69,75,79}$ The peaks positions agree with the literature on SERS of DNA and DNA bases, while the relative enhancement intensities of the single peaks turns out to be substrate-dependent (materials, shapes). ${ }^{66,67,69,70,75}$ The surface blocking with 6-MHO (blue curve) does not shift the DNA vibrations, neither provides new peaks to the spectrum, but only yields some increase of the continuum background. A slight decrease of the DNA peaks intensity is observed. Since the MOH has a thiol group as well as the aptamer, it is in competition with the aptamer, and some could be removed from the gold surface. This could explain the intensity decrease. A good reproducibility of the DNA spectra is observed in terms of position of the peaks, intensity along the surface, etc. independently from the nanoantennas shapes and sizes. Spectra acquired on the flat gold surfaces, present on the same substrate and undergone to the same binding process, display only a flat background with no signal from DNA. The noise level associated to this background can be used to provide a lower limit estimate of the SERS enhancement. On the nanoantenna dimers we find enhancements higher than $10^{3}$, in agreement with what measured using dyes molecular probes. ${ }^{41}$

After MnSOD deposition, it is possible to detect locally some high SERS signal of the biomarker using the nanorod dimers substrate. 3 (red line) shows the SERS spectrum 
of MnSOD at a concentration of $10^{-7} \mathrm{M}$ compared to the signal of the functionalization layer (DNA + MOH). Together with the DNA signal (highlighted by the dashed boxes) new, intense vibrational peaks are observed at 1140 and $1150 \mathrm{~cm}^{-1}$ (aliphatic side chain $\mathrm{CH}_{3}$ bending), $1350 \mathrm{~cm}^{-1}\left(\mathrm{CH}_{2}\right.$ bending), $1460 \mathrm{~cm}^{-1}\left(\mathrm{CH}_{2}\right.$ nonaromatic bending), $1536 \mathrm{~cm}^{-1}$ and in the $1605-1650 \mathrm{~cm}^{-1}$ range (Phe, and Amide I region). Most of these peaks, together with other smaller contributions, match the vibrations of MnSOD already detected on lyophilized powder with conventional Raman techniques (see SI, Erreur ! Source du renvoi introuvable.). ${ }^{80}$ These results prove that the functionalized nanostructures allow to detect MnSOD via its enhanced Raman signal. The amide III bands are also observable between 1240 and $1300 \mathrm{~cm}^{-1}$.

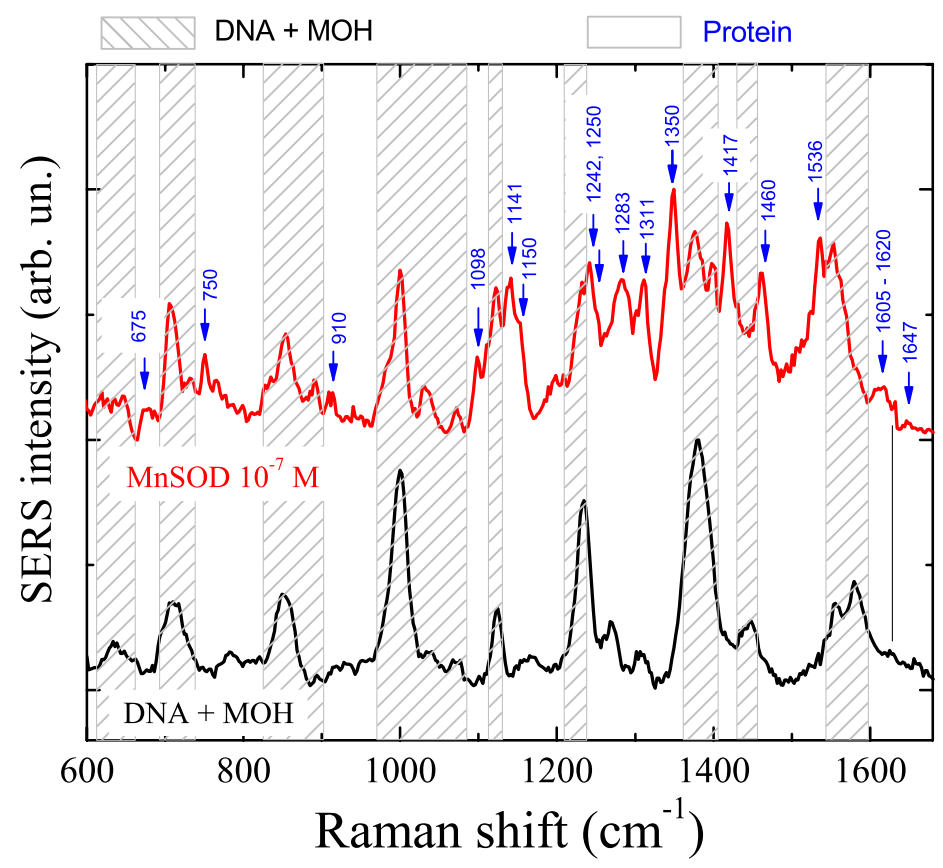

Figure 3: (red line) SERS spectrum of MnSOD after incubation of the functionalized nanoantenna dimers sample with a solution with protein at concentration $10^{-7} \mathrm{M}\left(\lambda_{\mathrm{exc}}=785 \mathrm{~nm}\right)$. In addition to the spectral features (highlighted by the dashed boxes) arising from of the SERS of the functionalization layer (black 
line), we can see new peaks due to the protein whose position is labeled. Spectra are shown after the continuum background removal and offset vertically for clarity reasons.

However, such a strong signal from the protein has not been observed on the whole nanoantennas surface. This can be attributed to the fact that the MnSOD is not distributed homogeneously and its adsorption is non-uniform on the nanobiosensor surface. In order to get rid of such problem and be able to detect the MnSOD up-take on the surface especially at the lowest concentrations, we have established a measurement protocol in which 10 spectra are recorded at different points of the nanobiosensor surface and an average spectrum is calculated, giving information on the presence of the MnSOD. Measurement points were chosen randomly on all surface patterns.

\section{Sensitivity}

Any assessment is carried out using, as reference, the SERS signal of MnSOD in the spectral bands highlighted in Figure . Experiments to establish the limit of detection (LOD) have been done at decreasing concentrations from $1 \mu \mathrm{M}$ to $10 \mathrm{nM}$ using both nanorods (Figure 4a) and nanocylinders (Figure 4b). To get rid of non homogenous absorption of the aptamer on the nanoantennas (which would have straightforward consequences on the quantity of MnSOD captured by the nanobiosensor) we plot the spectra normalized to the most intense peak of DNA at $1380 \mathrm{~cm}^{-1}$. In such a way spectra on different substrates, acquired under different experimental conditions can be compared. MnSOD is clearly detected down to $100 \mathrm{nM}$ using both substrates. The protein SERS spectra show new features with respect to the functionalization layer (also shown in the figures). Main differences arise in the $1490-1670 \mathrm{~cm}^{-1}$ 
region, where the characteristic bands of aromatic aminoacids (Tyr, Phe), Amide II and Amide I occur. The signal in this region is much broader than the one of DNA with the appearance of peaks at 1490, 1540, 1610, $1650 \mathrm{~cm}^{-1}$. Less intense spectral signatures of MnSOD are detected also at 1350, 1155 and $754 \mathrm{~cm}^{-1}$ (on both substrates) and at 935,905 and $679 \mathrm{~cm}^{-1}$ (using the nanorods). This could be given to the different enhancement that different vibrational modes can experience, especially when the chemical enhancement plays a role. ${ }^{81}$ All these new bands are comparable to the ones observed in the case of high SERS enhancement (Figure 3) and, even if their relative intensity can be different, the MnSOD signal turns out to be well reproducible with the different concentrations and substrates. However, all these differences are smoothed on the average spectrum. This is confirmed since whatever the nanostructure or the excitation wavelength used, the SERS signal did not exhibit relevant changes in the Raman modes (position as well as relative intensity) with the MnSOD concentration. Reducing the MnSOD concentration to $10 \mathrm{nM}$, we still observe a clear increase of signal in the $1490-1670 \mathrm{~cm}^{-1}$ region (Figure $4 \mathrm{~b}$ ), proving that our nanobiosensor can attain such LOD. However, at such low concentration, we observed a clear vibrational fingerprint of the protein only using the nanocylinders substrate. In this experimental condition, we believe that the inhomogeneity of the MnSOD uptake starts playing an important role, suggesting that longer incubation times, more dense surface sampling or simply nanoantennas with higher enhancement factor are needed to improve sensitivity and reproducibility. 

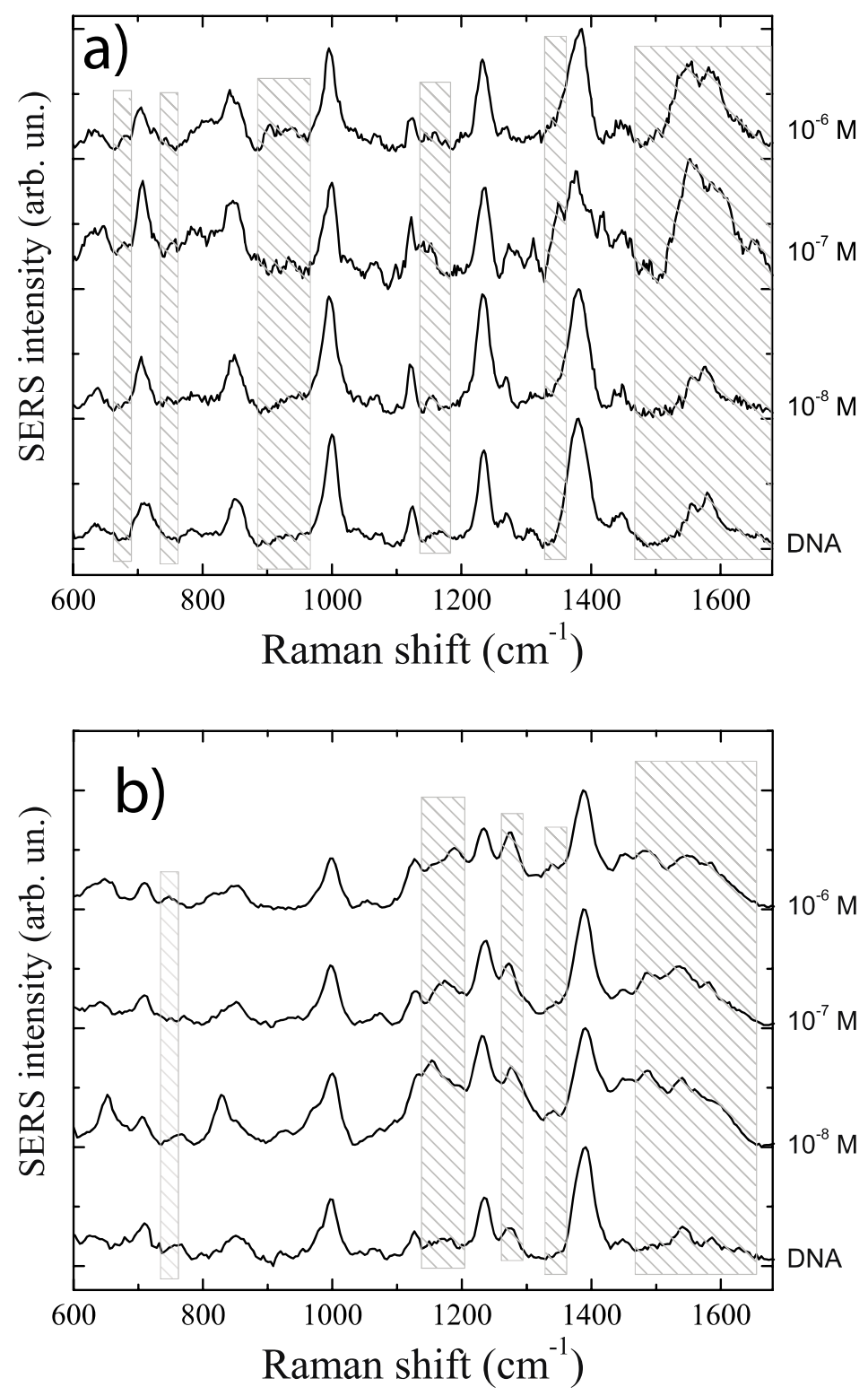

Figure 4: SERS detection of MnSOD at concentrations of $10^{-6}, 10^{-7}, 10^{-8} \mathrm{M}$ using (a) functionalized nanorods dimers $\left(\lambda_{\text {exc }}=785 \mathrm{~nm}\right)$ and (b) functionalized nanocylinders with $140 \mathrm{~nm}$ diameter $\left(\lambda_{\mathrm{exc}}=660 \mathrm{~nm}\right)$. For each SERS substrate the reference SERS spectrum of the functionalization layer is also shown (DNA). The dashed boxes highlight the spectral zones in which the protein provides strongest contribution. The spectra are plotted after background subtraction and offset vertically for clarity. 
Moreover, in the case of the nanorod dimers, one can notice that the MnSOD signal is lower than in the case of the nanocylinders and the specific MnSOD features are less visible. First, the geometry of the nanostructure can influence the orientation and position of the aptamer on the nanostructure surface and then on its ability to detect the MnSOD. Second, the enhancement comes essentially from the gap between two nanorods whereas it comes from a larger surface with the nanocylinders. As a consequence, there are less active sites on the dimer surface than on the nanocylinder surface. The probability of capture of MnSOD is lower, and then it is more difficult to observe the MnSOD at lower concentration. Both effects can affect the SERS signal of the MnSOD and explain the differences observable with both geometries.

Our data suggest that we reach a saturation of the SERS surface for a concentration of $100 \mathrm{nM}$. This saturation level can be explained by the effective surface with which the MnSOD can interact. Indeed, from QCM we have been able to calculate that the $\mathrm{Kd}$ is in the range of $300 \mathrm{nM}$. This value means that half of the aptamers are occupied by a MnSOD protein at this concentration on a flat gold surface. But the surface available with a nanorod dimers or a nanocylinder on a glass substrate is twice or four times lower than the one of the equivalent flat gold surface, respectively. The number of available aptamers is then lower on the SERS substrates and the SERS surfaces are saturated for lower MnSOD concentration as observed experimentally.

\section{Selectivity}

In order to demonstrate the specificity of the nanobiosensor we have performed two control experiments. First, we check that proteins different from MnSOD do not give any SERS signal. Second, we show that the blocking agent $(\mathrm{MOH})$ alone is not capable to bind MnSOD. For the first test we incubate the nanoantennas functionalized with aptamer in a Bovine Serum Albumin (BSA) solution at a 
concentration of $10 \mu \mathrm{M}$, i.e. 100 times higher that the one at which we detect MnSOD. As shown in Figure, no significant changes are observable between the SERS signal given by the nanobiosensor after incubation with BSA (red line) and the SERS signal from the aptamer+MOH layer only (black line), even if the BSA concentration is high enough to saturate the sensor surface. If any interaction had occurred between the BSA and the aptamer, we should have observed some additional signal on the SERS spectrum in spectral zones, such as Amide I, Amide II, Amide III, aromatic amino-acids, specific of proteins, where the contribution of MnSOD is observed (dashed boxes in Figure 5), which is not the case.

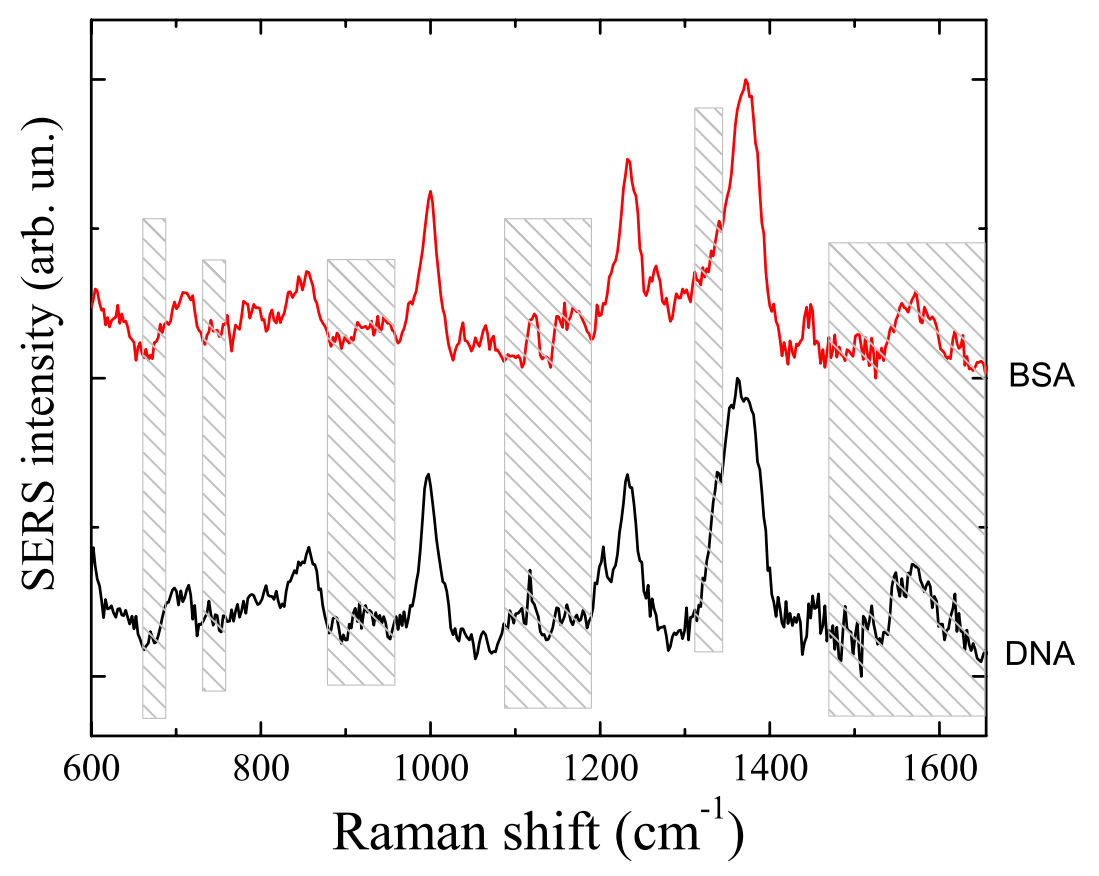

Figure 5: Negative control experiment. The SERS spectrum on gold nanorods dimers $\left(\lambda_{\text {exc }}=785 \mathrm{~nm}\right)$ after incubation with BSA $10^{-5} \mathrm{M}$ (red line) does not show specific differences with respect to the SERS from the aptamer+MOH layer (black line) in the spectral zones in which the proteins feature their vibrational signal (dashed boxes). Spectra are offset vertically for clarity reasons. 
This demonstrates that the non specific binding does not occur with using MnSOD-aptamers to functionalize the nanoantennas and, in addition, that no unspecific binding occurs between BSA and the 6-MOH blocking agent. Same negative results (see SI, S-5, Figure S3) are found when we functionalize the nanoantennas with 6-MOH only and incubate with MnSOD. This second control experiment excludes, as for BSA, any strong unspecific interaction between the blocking layer (6-MOH), and the MnSOD and supports the fact that the capture of the MnSOD is only due to the presence of aptamers on the surface without any significant role played by 6-MOH.

The previous results demonstrate that the designed nanobiosensor is specific and that the MnSOD can be detected at low concentrations.

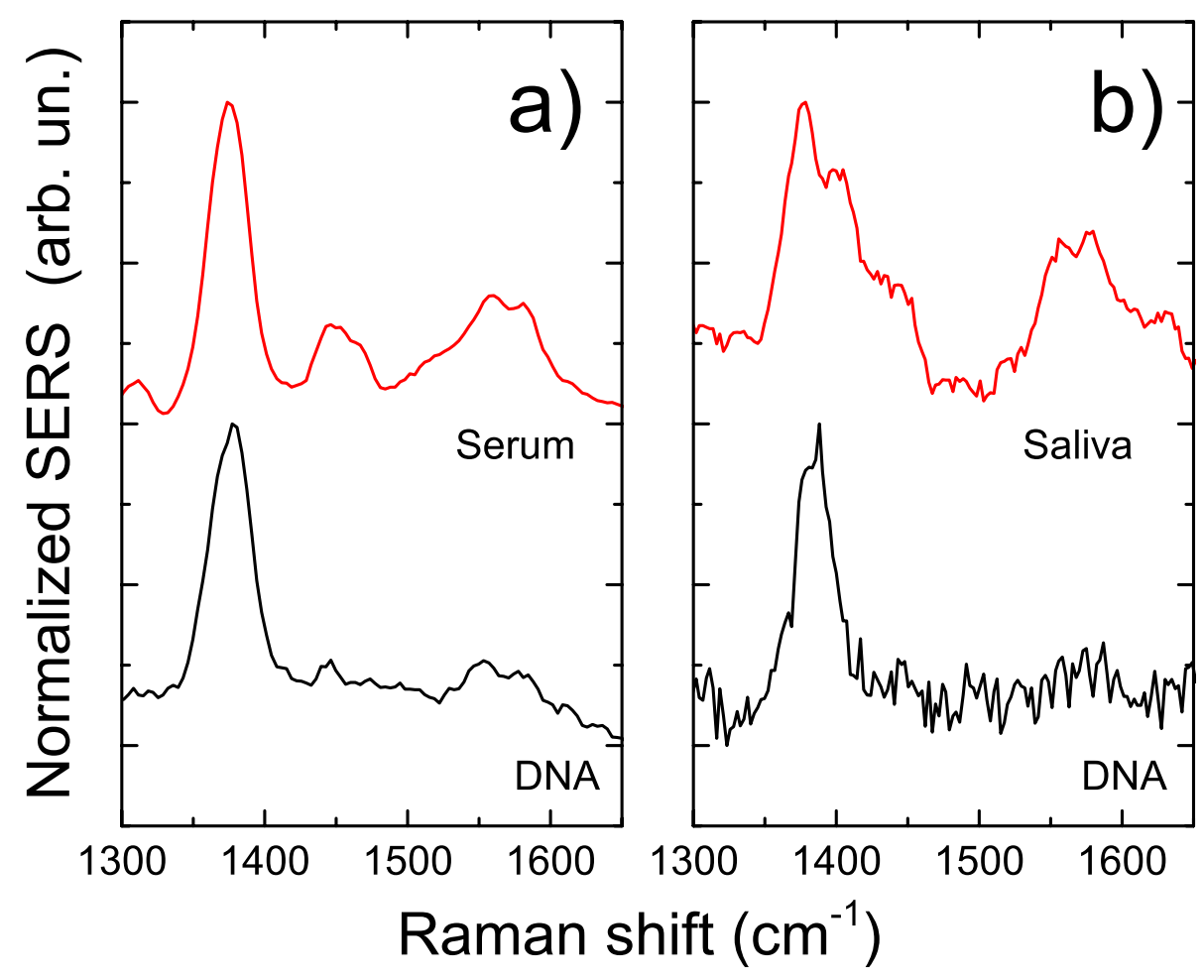


Figure 6: MnSOD detection in body fluids. (a, red line) SERS signal of MnSOD in serum at $10^{-8} \mathrm{M}$ compared that of the aptamer (black line) using gold nanocylinders $\left(\lambda_{\text {exc }}=660 \mathrm{~nm}\right) .\left(\mathrm{b}\right.$, red line). Spectrum of MnSOD in saliva at $10^{-8} \mathrm{M}$ compared that of aptamer (black line) on gold nanorods dimers $\left(\lambda_{\mathrm{exc}}=785 \mathrm{~nm}\right)$.

\section{Detection in body fluids}

For further evaluation of the nanobiosensor performances, the detection of MnSOD in complex biological media (saliva and sera samples) has been tested. The MnSOD concentrations are $10^{-8} \mathrm{M}$ in both saliva and serum, as measured by ELISA. The protocol for the MnSOD deposition is the same as the one used for the pure MnSOD except that the pure MnSOD solution is replaced by the body fluid.

Figure 6a shows the SERS signal from one sample of serum (a, red line) using nanocylinders and excitation wavelength at $660 \mathrm{~nm}$ and (Figure 6b, red line) from one sample of saliva using nanorod dimers and excitation at $785 \mathrm{~nm}$. The black lines in (a, b) represent the reference spectra of the functionalization layer (aptamer and 6-MHO) before the deposition of the body fluids. In the spectra of serum and saliva strongest signal increase is observed in the $1450-1650 \mathrm{~cm}^{-1}$ band, comparable to what observed with pure MnSOD solutions (Figure 4). One can also notice a slight difference between both spectra since a band located at $1400 \mathrm{~cm}^{-1}$ is only observable on the saliva spectrum. However, this band can be assigned to the COO- streching (see SI, Table S1 and Figure 3) and is due to the presence of the MnSOD. Such small variations between these spectra can be explained by the protein environment. saliva and serum are complex media that can slightly affect the protein structure and as a consequence the SERS signal. In the case of nonspecific binding of other proteins present in the body fluids, we should observe some other bands due to different 
spectral signatures compared to the MnSOD one. The Raman will not be systematically at the same position and with similar relative intensity whatever the media used (pure MnSOD or body fluids). Since both body fluids contain the same MnSOD concentration, we could also compare the dimers and nanocylinders in terms of detection. In both cases, we were able to observe the MnSOD signal, and thus both nanostructures have similar efficiency for the detection in body fluids. These results, together with the specific SERS fingerprint observation on the pure MnSOD solutions and the specificity tests, support the conclusion that our nanobiosensor can detect MnSOD in body fluids at a concentration of $10 \mathrm{nM}$, whatever the nanostructures used.

Even if we have demonstrated the proof of concept of our nanobiosensor in the $\mathrm{nM}$ range, it could be possible to improve the detection and to decrease its limit of detection. This could be done by changing the nanostructure geometry to increase the SERS of efficiency. For example, it has been demonstrated that dimers of gold nanorods with a slanted gap provides an improvement of the enhancement factor by 2 orders of magnitude compared to the rounded shape use in this paper. ${ }^{79}$ Another example is given by the change of the adhesion layer ( $\mathrm{Cr}$ or $\mathrm{Ti}$ ) between the gold nanostructures and the dielectric substrate by an adhesion layer of (3mercaptopropyl)- trimethoxysilane (MPTMS). In this case, the LSPR damping is decreased that induces an improvement of the SERS signal by a factor $10.80,81$ Thus, by combining these effects, we assume that we can gain several orders of magnitude on the detection limits.

Furthermore, this concept of SERS sensor could be largely applied in other fields such as the observation of proteins and of their change of conformation due to environmental modification (phosphorylation, denaturation, ...) or as the monitoring of chemical reaction (catalytic reaction, ...) in solution or in air. Thanks to the highly 
reproducible SERS substrate produced by EBL, the Raman signal will be reliable which open the way to the quantification of the monitored biological or chemical processes and to the comparison between several processes. Moreover, the use of a bioreceptor could avoid the interaction of the analytes with the gold surface and thus the potential perturbation of this surface on the analyte conformation, especially in the case of proteins.

\section{Conclusion}

We have developed a high sensitive, high specific SERS nanobiosensor, that uses EBL optical nanoantennas functionalized with DNA aptamers for specific detection of molecular biomarkers at nanomolar concentrations. Both individual nanocylinders and $20 \mathrm{~nm}$ gap nanorods dimers have proved the capability to detect MnSOD in pure solutions and, most importantly, in body fluids such as serum and saliva. Our measurements suggest that the use of optimized SERS substrate (e.g., dimers with nm-gap dimensions, or nanostars) can improve the sensitivity of our sensor and that detection limit lower than the $\mathrm{nM}$ can be attained, which is a competitive advantage for the biomarker detection.

\section{Experimental section}

Materials: Human Manganese SuperOxide Dismutase (MnSOD) was provided by Abfrontier. Potassium chloride $(\mathrm{KCl})$, trizma base, 6-mercapto-1-hexanol (MHO), magnesium chloride hexahydrate $\left(\mathrm{MgCl}_{2}\right)$, ammonium hydroxide solution $28 \%-30 \%$ $\left(\mathrm{NH}_{4} \mathrm{OH}\right)$, ethanol and HEPES buffer were purchased from Sigma-Aldrich. 2.0 M triethylamine acetate buffer, trifluoroacetic acid, acetonitrile were provided by Applied Biosystems. MilliQ water (18.2 M $\Omega$ ) was used to prepare all solutions. 
MnSOD aptamers. Thiolated aptamers were prepared on a 3400 DNA synthesizer (Applied Biosystems). Standard DNA phosphoramidites, 5'-thiol-modifier C6-CE phosphoramidite and other reagents required for solid phase oligonucleotide synthesis were purchased from Link Technologies. After synthesis, oligonucleotide was cleaved from the solid support and deprotected by reaction with concentrated $\mathrm{NH}_{4} \mathrm{OH}$ during $15 \mathrm{~h}$ at $+55^{\circ} \mathrm{C}$ while stirring. Oligonucleotide purification cartridges (Applied Biosystems) were used for the following purification of thiolated aptamer. The concentration of pure oligonucleotide was determined by measuring absorbance at $\lambda=260 \mathrm{~nm}$ on ND 1000 Spectrophotometer. This Aptamer is specific to the MnSOD protein.

QCM experiments. QCM-D experiments were performed using a Q-Sense E4 unit (Q Sense $A B$, Sweden) and the shifts in frequency $(\Delta \mathrm{f})$ were monitored in real time. The mounted Au-quartz crystal (QSX301, Q-Sense AB) is inserted inside a flow cell (QFM401, Q-Sense) in order to inject the different solutions of interest. The modifications of QCM electrodes and subsequent washings were performed inside of the flow cell. All QCM measurements were undertaken at $23{ }^{\circ} \mathrm{C}$ at a flow rate of 100 $\mu \mathrm{L} / \min$.

Optical nanoantennas. Optical nanoantennas are produced by Electron beam lithography on a glass substrate with a $3 \mathrm{~nm}$ adhesion layer of chromium for the 140 nm nanocylinders and on a $\mathrm{CaF}_{2}$ (100) substrate with a $3 \mathrm{~nm}$ adhesion layer of titanium for the nanorod dimers, as described in refs. ${ }^{41,62}$ 
SERS spectroscopy. We use an Xplora microspectrometer equipped with two different excitation lasers (660 and $785 \mathrm{~nm})$ and with a peltier-cooled CCD for signal detection. SERS measurements on the nanorods dimers and on the 200nm diameter nanocylinders are carried out at $785 \mathrm{~nm}$, while $660 \mathrm{~nm}$ excitation is used for the $140 \mathrm{~nm}$ diameter nanocylinders. A $100 \mathrm{X}$ magnification microscope objective with a numerical aperture of 0.9 is used for sample excitation and collection of the backscattered light. For both excitation wavelengths, the laser spot is nearly diffraction limited with a diameter around $1 \mu \mathrm{m}$. The laser power on the sample is always less than $1 \mathrm{~mW}$ to prevent sample degradation, while the polarization is parallel to the gold dimer axis. Acquisition time of each spectrum is 60 s. To have a better observation of the spectra, a base line correction has been done using the Raman spectrometer software and the spectra have been normalized with regards to the most intense Raman band.

\section{Acknowledgement}

Authors acknowledge the European project Nanoantenna (HEALTH-F5-2009241818) for the financial support. PGG, BF and CD'A acknowledge MIUR (PRIN 2008J858Y7 and PON01_01322 PANREX)

\section{REFERENCES}

\section{Uncategorized References}

1. Lequin, R. M., Enzyme immunoassay (EIA)/enzyme-linked immunosorbent assay (ELISA). Clinical chemistry 2005, 51, 2415-2418.

2. Ronkainen, N. J.; Halsall, H. B.; Heineman, W. R., Electrochemical biosensors. Chemical Society Reviews 2010, 39, 1747-1763. 
3. Ligler, F. S., Perspective on Optical Biosensors and Integrated Sensor Systems. Analytical Chemistry 2008, 81, 519-526.

4. Fan, X.; White, I. M.; Shopova, S. I.; Zhu, H.; Suter, J. D.; Sun, Y., Sensitive optical biosensors for unlabeled targets: A review. Analytica Chimica Acta 2008, 620, 8-26.

5. Borisov, S. M.; Wolfbeis, O. S., Optical biosensors. Chemical reviews 2008, 108, 423-461.

6. Ligler, F. S.; Sapsford, K. E.; Golden, J. P.; Shriver-Lake, L. C.; Taitt, C. R.; Dyer, M. A.; Barone, S.; Myatt, C. J., The array biosensor: portable, automated systems. Analytical sciences : the international journal of the Japan Society for Analytical Chemistry 2007, 23, 5-10.

7. Taitt, C. R.; Anderson, G. P.; Ligler, F. S., Evanescent wave fluorescence biosensors. Biosensors and Bioelectronics 2005, 20, 2470-2487.

8. Homola, J., Present and future of surface plasmon resonance biosensors. Anal Bioanal Chem 2003, 377, 528-539.

9. Arlett, J. L.; Myers, E. B.; Roukes, M. L., Comparative advantages of mechanical biosensors. Nature nanotechnology 2011, 6, 203-215.

10. Tamayo, J.; Kosaka, P. M.; Ruz, J. J.; San Paulo, Á.; Calleja, M., Biosensors based on nanomechanical systems. Chemical Society Reviews 2013, 42, 1287-1311.

11. O'sullivan, C. K.; Guilbault, G. G., Commercial quartz crystal microbalancestheory and applications. Biosensors and bioelectronics 1999, 14, 663-670.

12. Ligler, F. S.; Taitt, C. R.; Shriver-Lake, L. C.; Sapsford, K. E.; Shubin, Y.; Golden, J. P., Array biosensor for detection of toxins. Anal Bioanal Chem 2003, 377, 469-77.

13. Jia, K.; Eltzov, E.; Toury, T.; Marks, R. S.; Ionescu, R. E., A lower limit of detection for atrazine was obtained using bioluminescent reporter bacteria via a lower incubation temperature. Ecotoxicology and Environmental Safety 2012, 84, 221-226.

14. Garai-Ibabe, G.; Grinyte, R.; Golub, E. I.; Canaan, A.; de la Chapelle, M. L.; Marks, R. S.; Pavlov, V., Label free and amplified detection of cancer marker EBNA1 by DNA probe based biosensors. Biosensors and Bioelectronics 2011, 30, 272-275.

15. Tombelli, S.; Minunni, M.; Mascini, M., Analytical applications of aptamers. Biosensors and Bioelectronics 2005, 20, 2424-2434.

16. Zhang, D.; Xie, Y.; Mrozek, M. F.; Ortiz, C.; Davisson, V. J.; Ben-Amotz, D., Raman detection of proteomic analytes. Analytical chemistry 2003, 75, 5703-5709.

17. Fang, Z.; Ge, C.; Zhang, W.; Lie, P.; Zeng, L., A lateral flow biosensor for rapid detection of DNA-binding protein c-jun. Biosensors and Bioelectronics 2011, 27, 192-196.

18. Miao, Y.; Cui, T.; Leng, F.; Wilson, W. D., Inhibition of high-mobility-group A2 protein binding to DNA by netropsin: A biosensor-surface plasmon resonance assay. Analytical Biochemistry 2008, 374, 7-15.

19. Wu, L.; Zhang, Q.; Su, L.; Huang, M.; Zhao, J.; Yang, M., Effects of small molecular inhibitors on the binding between HIV-1 reverse transcriptase and DNA as revealed by SPR biosensor. Sensors and Actuators B: Chemical 2007, 122, 243-252.

20. Hong, S.-R.; Jeong, H.-D.; Hong, S., QCM DNA biosensor for the diagnosis of a fish pathogenic virus VHSV. Talanta 2010, 82, 899-903. 
21. Keefe, A. D.; Pai, S.; Ellington, A., Aptamers as therapeutics. Nat Rev Drug Discov 2010, 9, 537-550.

22. Ferraro, J. R., Introductory raman spectroscopy. Academic press: 2003.

23. David, C.; d'Andrea, C.; Lancelot, E.; Bochterle, J.; Guillot, N.; Fazio, B.; Maragò, O. M.; Sutton, A.; Charnaux, N.; Neubrech, F., Raman and IR spectroscopy of manganese superoxide dismutase, a pathology biomarker. Vibrational Spectroscopy 2012, 62, 50-58.

24. Le Ru, E.; Etchegoin, P., Principles of Surface-Enhanced Raman Spectroscopy: and related plasmonic effects. Elsevier: 2008.

25. David, C.; Guillot, N.; Shen, H.; Toury, T.; de la Chapelle, M. L., SERS detection of biomolecules using lithographed nanoparticles towards a reproducible SERS biosensor. Nanotechnology 2010, 21, 475501.

26. Hao, E.; Schatz, G. C., Electromagnetic fields around silver nanoparticles and dimers. The Journal of chemical physics 2004, 120, 357-366.

27. Fazio, B.; D’Andrea, C.; Bonaccorso, F.; Irrera, A.; Calogero, G.; Vasi, C.; Gucciardi, P. G.; Allegrini, M.; Toma, A.; Chiappe, D., Re-radiation enhancement in polarized surface-enhanced resonant Raman scattering of randomly oriented molecules on self-organized gold nanowires. ACS Nano 2011, 5, 5945-5956.

28. Le Ru, E.; Blackie, E.; Meyer, M.; Etchegoin, P. G., Surface enhanced Raman scattering enhancement factors: a comprehensive study. The Journal of Physical Chemistry C 2007, 111, 13794-13803.

29. Le Ru, E.; Meyer, M.; Etchegoin, P., Proof of single-molecule sensitivity in surface enhanced Raman scattering (SERS) by means of a two-analyte technique. The Journal of Physical Chemistry B 2006, 110, 1944-1948.

30. Le Ru, E. C.; Etchegoin, P. G., Single-molecule surface-enhanced Raman spectroscopy. Annual review of physical chemistry 2012, 63, 65-87.

31. Anker, J. N.; Hall, W. P.; Lyandres, O.; Shah, N. C.; Zhao, J.; Van Duyne, R. P., Biosensing with plasmonic nanosensors. Nature materials 2008, 7, 442-453.

32. Han, X. X.; Zhao, B.; Ozaki, Y., Surface-enhanced Raman scattering for protein detection. Anal Bioanal Chem 2009, 394, 1719-1727.

33. Tripp, R. A.; Dluhy, R. A.; Zhao, Y., Novel nanostructures for SERS biosensing. Nano Today 2008, 3, 31-37.

34. Guillot, N.; de la Chapelle, M. L., Lithographied nanostructures as nanosensors. Journal of Nanophotonics 2012, 6, 064506-1-064506-28.

35. Bantz, K. C.; Meyer, A. F.; Wittenberg, N. J.; Im, H.; Kurtuluş, Ö.; Lee, S. H.; Lindquist, N. C.; Oh, S.-H.; Haynes, C. L., Recent progress in SERS biosensing. Physical Chemistry Chemical Physics 2011, 13, 11551-11567.

36. Douglas, P.; Stokes, R. J.; Graham, D.; Smith, W. E., Immunoassay for P38 MAPK using surface enhanced resonance Raman spectroscopy (SERRS). Analyst 2008, 133, 791-796.

37. Stokes, R. J.; McBride, E.; Wilson, C. G.; Girkin, J. M.; Smith, W. E.; Graham, D., Surface-enhanced Raman scattering spectroscopy as a sensitive and selective technique for the detection of folic acid in water and human serum. Applied spectroscopy 2008, 62, 371-6.

38. Grubisha, D. S.; Lipert, R. J.; Park, H.-Y.; Driskell, J.; Porter, M. D., Femtomolar detection of prostate-specific antigen: an immunoassay based on surface- 
enhanced Raman scattering and immunogold labels. Analytical chemistry 2003, 75, 5936-5943.

39. Guillot, N.; Shen, H.; Frémaux, B.; Peron, O.; Rinnert, E.; Toury, T.; De La Chapelle, M. L., Surface enhanced Raman scattering optimization of gold nanocylinder arrays: Influence of the localized surface plasmon resonance and excitation wavelength. Applied Physics Letters 2010, 97, 023113.

40. McFarland, A. D.; Young, M. A.; Dieringer, J. A.; Van Duyne, R. P., Wavelength-scanned surface-enhanced Raman excitation spectroscopy. The Journal of Physical Chemistry B 2005, 109, 11279-11285.

41. D'Andrea, C.; Bochterle, J.; Toma, A.; Huck, C.; Neubrech, F.; Messina, E.; Fazio, B.; Marago, O. M.; Di Fabrizio, E.; Lamy de La Chapelle, M., Optical nanoantennas for multiband surface-enhanced infrared and Raman spectroscopy. ACS nano 2013, 7, 3522-3531.

42. Guillot, N.; de la Chapelle, M. L., The electromagnetic effect in surface enhanced Raman scattering: Enhancement optimization using precisely controlled nanostructures. Journal of Quantitative Spectroscopy and Radiative Transfer 2012, 113, 2321-2333.

43. Shafer-Peltier, K. E.; Haynes, C. L.; Glucksberg, M. R.; Van Duyne, R. P., Toward a glucose biosensor based on surface-enhanced Raman scattering. Journal of the American Chemical Society 2003, 125, 588-593.

44. Stuart, D. A.; Yuen, J. M.; Shah, N.; Lyandres, O.; Yonzon, C. R.; Glucksberg, M. R.; Walsh, J. T.; Van Duyne, R. P., In vivo glucose measurement by surface-enhanced Raman spectroscopy. Analytical chemistry 2006, 78, 7211-7215.

45. Zhang, X.; Young, M. A.; Lyandres, O.; Van Duyne, R. P., Rapid detection of an anthrax biomarker by surface-enhanced Raman spectroscopy. Journal of the American Chemical Society 2005, 127, 4484-4489.

46. Das, G.; Mecarini, F.; De Angelis, F.; Prasciolu, M.; Liberale, C.; Patrini, M.; Di Fabrizio, E., Attomole (amol) myoglobin Raman detection from plasmonic nanostructures. Microelectronic Engineering 2008, 85, 1282-1285.

47. De Angelis, F.; Gentile, F.; Mecarini, F.; Das, G.; Moretti, M.; Candeloro, P.; Coluccio, M.; Cojoc, G.; Accardo, A.; Liberale, C.; Proietti Zaccaria, R.; Perozziello, G.; Tirinato, L.; Toma, A.; Cuda, G.; Cingolani, R.; Di Fabrizio, E., Breaking the diffusion limit with super-hydrophobic delivery of molecules to plasmonic nanofocusing SERS structures. Nature Photonics 2011, 5, 682-687.

48. Cho, H.; Baker, B. R.; Wachsmann-Hogiu, S.; Pagba, C. V.; Laurence, T. A.; Lane, S. M.; Lee, L. P.; Tok, J. B.-H., Aptamer-based SERRS sensor for thrombin detection. Nano letters 2008, 8, 4386-4390.

49. Li, M.; Zhang, J.; Suri, S.; Sooter, L. J.; Ma, D.; Wu, N., Detection of adenosine triphosphate with an aptamer biosensor based on surface-enhanced Raman scattering. Analytical chemistry 2012, 84, 2837-2842.

50. Huh, Y. S.; Erickson, D., Aptamer based surface enhanced Raman scattering detection of vasopressin using multilayer nanotube arrays. Biosensors and Bioelectronics 2010, 25, 1240-1243.

51. Borrelli, A.; Schiattarella, A.; Bonelli, P.; Tuccillo, F. M.; Buonaguro, F. M.; Mancini, A., The Functional Role of MnSOD as a Biomarker of Human Diseases and Therapeutic Potential of a New Isoform of a Human Recombinant MnSOD. BioMed research international 2014, 2014. 
52. Epperly, M. W.; Defilippi, S.; Sikora, C.; Gretton, J.; Greenberger, J. S., Radioprotection of lung and esophagus by overexpression of the human manganese superoxide dismutase transgene. Military Medicine 2002, 167, 71-73.

53. Mukhopadhyay, S.; Das, S. K.; Mukherjee, S., Expression of Mn-Superoxide Dismutase Gene in Nontumorigenic and Tumorigenic Human Mammary Epithelial Cells. Journal of Biomedicine and Biotechnology 2004, 4, 195-202.

54. Li, J. J.; Oberley, L. W.; St Clair, D. K.; Ridnour, L. A.; Oberley, T. D., Phenotypic changes induced in human breast cancer cells by overexpression of manganese-containing superoxide dismutase. Oncogene 1995, 10, 1989-2000.

55. Church, S. L.; Grant, J. W.; Ridnour, L. A.; Oberley, L. W.; Swanson, P. E.; Meltzer, P. S.; Trent, J. M., Increased manganese superoxide dismutase expression suppresses the malignant phenotype of human melanoma cells. Proceedings of the National Academy of Sciences 1993, 90, 3113-3117.

56. Zhong, W.; Oberley, L. W.; Oberley, T. D.; St Clair, D. K., Suppression of the malignant phenotype of human glioma cells by overexpression of manganese superoxide dismutase. Oncogene 1997, 14, 481-90.

57. McGlynn, K. A.; Tarone, R. E.; El-Serag, H. B., A comparison of trends in the incidence of hepatocellular carcinoma and intrahepatic cholangiocarcinoma in the United States. Cancer epidemiology, biomarkers \& prevention : a publication of the American Association for Cancer Research, cosponsored by the American Society of Preventive Oncology 2006, 15, 1198-203.

58. Tamai, T.; Uto, H.; Takami, Y.; Oda, K.; Saishoji, A.; Hashiguchi, M.; Kumagai, K.; Kure, T.; Mawatari, S.; Moriuchi, A., et al., Serum manganese superoxide dismutase and thioredoxin are potential prognostic markers for hepatitis $\mathrm{C}$ virus-related hepatocellular carcinoma. World journal of gastroenterology : WJG 2011, 17, 4890-8.

59. Kawaguchi, T.; Suzuki, K.-i.; Matsuda, Y.; Nishiura, T.; Uda, T.; Ono, M.; Sekiya, C.; Ishikawa, M.; Iino, S.; Endo, Y., et al., Serum-manganese-superoxide dismutase: normal values and increased levels in patients with acute myocardial infarction and several malignant diseases determined by an enzyme-linked immunosorbent assay using a monoclonal antibody. Journal of Immunological Methods 1990, 127, 249-254.

60. Ishikawa, M.; Yaginuma, Y.; Hayashi, H.; Shimizu, T.; Endo, Y.; Taniguchi, N., Reactivity of a monoclonal antibody to manganese superoxide dismutase with human ovarian carcinoma. Cancer research 1990, 50, 2538-42.

61. Schadendorf, D.; Zuberbier, T.; Diehl, S.; Schadendorf, C.; Czarnetzki, B. M., Serum manganese superoxide dismutase is a new tumour marker for malignant melanoma. Melanoma research 1995, 5, 351-3.

62. Kessentini, S.; Barchiesi, D.; D'Andrea, C.; Toma, A.; Guillot, N.; Di Fabrizio, E.; Fazio, B.; Maragó, O. M.; Gucciardi, P. G.; Lamy de la Chapelle, M., Gold Dimer Nanoantenna with Slanted Gap for Tunable LSPR and Improved SERS. The Journal of Physical Chemistry C 2014, 118, 3209-3219.

63. Mahajan, S.; Cole, R. M.; Speed, J. D.; Pelfrey, S. H.; Russell, A. E.; Bartlett, P. N.; Barnett, S. M.; Baumberg, J. J., Understanding the Surface-Enhanced Raman Spectroscopy "Background" J. Phys. Chem. C 2010, 114, 7242 - 7250. 
64. Imura, K.; Okamoto, H., Properties of Photoluminescence from Single Gold Nanorods Induced by Near-Field Two-Photon Excitation. Journal of Physical Chemistry C 2009, 113, 11756-11759.

65. Gee, A. R.; O'Shea, D. C.; Cummins, H. Z., Raman scattering and fluorescence in calcium fluoride. Solid State Communications 1966, 4, 43-46.

66. Pagba, C. V.; Lane, S. M.; Wachsmann - Hogiu, S., Raman and surface enhanced Raman spectroscopic studies of the 15 - mer DNA thrombin - binding aptamer. Journal of Raman Spectroscopy 2010, 41, 241-247.

67. Bi, L.; Rao, Y.; Tao, Q.; Dong, J.; Su, T.; Liu, F.; Qian, W., Fabrication of large-scale gold nanoplate films as highly active SERS substrates for label-free DNA detection. Biosensors and Bioelectronics 2013, 43, 193-199.

68. Jang, N. H., The coordination chemistry of DNA nucleosides on gold nanoparticles as a probe by SERS. BULLETIN-KOREAN CHEMICAL SOCIETY 2002, 23, 1790-1800.

69. Jang, N. H., SERS Analysis of Self-Assembled Monolayers of DNA Strands on Gold Surfaces. Bulletin of the Korean Chemical Society 2010, 31, 213-215.

70. Otto, C.; Van den Tweel, T.; De Mul, F.; Greve, J., Surface - enhanced Raman spectroscopy of DNA bases. Journal of Raman spectroscopy 1986, 17, 289298.

71. Duguid, J.; Bloomfield, V. A.; Benevides, J.; Thomas Jr, G. J., Raman spectroscopy of DNA-metal complexes. I. Interactions and conformational effects of the divalent cations: $\mathrm{Mg}, \mathrm{Ca}, \mathrm{Sr}, \mathrm{Ba}, \mathrm{Mn}, \mathrm{Co}, \mathrm{Ni}, \mathrm{Cu}, \mathrm{Pd}$, and $\mathrm{Cd}$. Biophysical journal 1993, 65, 1916-1928.

72. Giese, B.; McNaughton, D., Density functional theoretical (DFT) and surfaceenhanced Raman spectroscopic study of guanine and its alkylated derivatives Part 2: Surface-enhanced Raman scattering on silver surfaces. Physical Chemistry Chemical Physics 2002, 4, 5171-5182.

73. Rasmussen, A.; Deckert, V., Surface - and tip - enhanced Raman scattering of DNA components. Journal of Raman Spectroscopy 2006, 37, 311-317.

74. Marotta, N. E.; Beavers, K. R.; Bottomley, L. A., Limitations of surface enhanced Raman scattering in sensing DNA hybridization demonstrated by label-free DNA oligos as molecular rulers of distance-dependent enhancement. Analytical chemistry 2013, 85, 1440-1446.

75. Barhoumi, A.; Zhang, D.; Tam, F.; Halas, N. J., Surface-enhanced Raman spectroscopy of DNA. Journal of the American Chemical Society 2008, 130, 55235529.

76. Gearheart, L. A.; Ploehn, H. J.; Murphy, C. J., Oligonucleotide Adsorption to Gold Nanoparticles: A Surface-Enhanced Raman Spectroscopy Study of Intrinsically Bent DNA. Journal of of Physical Chemistry B 2001, 105, 12609-126015.

77. Muntean, C. M.; Dostál, L.; Misselwitz, R.; Welfle, H., DNA structure at low $\mathrm{pH}$ values, in the presence of Mn2+ ions: a Raman study. Journal of Raman Spectroscopy 2005, 36, 1047-1051.

78. Papadopoulou, E.; Bell, S. E., Label - Free Detection of Single - Base Mismatches in DNA by Surface - Enhanced Raman Spectroscopy. Angewandte Chemie International Edition 2011, 50, 9058-9061. 
79. Badr, Y.; Mahmoud, M., Effect of silver nanowires on the surface-enhanced Raman spectra (SERS) of the RNA bases. Spectrochimica Acta Part A: Molecular and Biomolecular Spectroscopy 2006, 63, 639-645.

80. David, C.; d'Andrea, C.; Lancelot, E.; Bochterle, J.; Guillot, N.; Fazio, B.; Maragò, O. M.; Sutton, A.; Charnaux, N.; Neubrech, F., et al., Raman and IR spectroscopy of manganese superoxide dismutase, a pathology biomarker. Vibrational Spectroscopy 2012, 62, 50-58.

81. Park, W.-H.; Kim, Z. H., Charge transfer enhancement in the SERS of a single molecule. Nano letters 2010, 10, 4040-4048. 